\title{
Low-cost VRML Based Architecture for Adaptive and User Customized Virtual Visits to Art Galleries
}

\author{
Martina Eckert ${ }^{1,}$ José Maria Cubero $^{2}$, Beatriz Sánchez ${ }^{1}$, Antonio Martín ${ }^{1}$, Luis Salgado ${ }^{2}$ \\ Affiliation ${ }^{1}$ : \\ Escuela Universitaria de Ingeniería Técnica de \\ Telecomunicación \\ Universidad Politécnica de Madrid \\ 28031 Madrid, Spain \\ martina.eckert@upm.es \\ Affiliation ${ }^{2}$ : \\ Escuela Técnica Superior de Ingenieros de \\ Telecomunicación \\ Universidad Politécnica de Madrid \\ 28040 Madrid, Spain \\ luis.salgado@gti.ssr.upm.es
}

\begin{abstract}
This paper presents an innovative procedure, capable to automatically generate virtual environments which are applicable to art galleries, museums or similar facilities, and suitable for web-based promotions of artists and their works. The presented system is part of the outcome of an interdisciplinary research for low-cost and platform independent solutions to combine art with virtual reality with the aim to promote artist works through new interaction metaphors which include dynamic 3D visualization of works of art with associated structured multimedia information.
\end{abstract}

In detail, we present the resulting low-cost and platformindependent VRML-based architecture for the automatic generation of user-customized virtual visits in 3D of art galleries based on XML descriptions.

As the VRML standard presents a lack of flexibility when customizing the scenarios according to special user interests, which could be different in every session, we propose a new strategy consisting in decoupling the associated multimedia information from the geometrical description of the presented artworks. This approach provides an external management of those contents, hence overcoming the identified limitation, while additionally increasing design efficiency and content adaptability.

Artworks, art galleries, adaptive virtual scenes, web technology, user customized virtual environment, low-cost, platform independent, VRML, XML, metaphors

\section{INTRODUCTION}

The use of Digital Image Processing is increasing in plastic and visual arts, since new technologies are constantly offering new possibilities for innovation. Now, the increasing multimedia capabilities of most common web browsers have enabled the incorporation of $3 \mathrm{D}$ graphics as a new accessible media for the general public. Web browsers can interpret and render interactive 3D scenes described in standard languages - such as VRML [1], [2] or X3D [3] thanks to publicly available plug-ins, thus enabling the generation of low-cost 3D graphics. The virtual worlds, generated according to real scenarios, provide the user experiences in a more interactive and immersive manner, and heterogeneous disciplines, above all the artistic sector, can take advantage of advances like the one presented in this document. It enables artists to push their creations with attractive and cheap instruments into the current collaborative and globalized social environment.

The work presented in this paper aims to facilitate a global access to art collections including their online accessible related materials, offering the possibility to create adaptive, user customized virtual visits. Thus we propose a low-cost VRML based architecture for the automatic generation of user-customized virtual environments based on XML descriptions [4]. Virtual scenes are thought to promote works of art which, with an appropriate set of metadata, allow their dynamic 3D visualization together with associated multimedia information. Geometrical descriptions of the modeled environment and artworks are decoupled to allow content adaptability with efficient model management. Also, the hierarchical access to multimedia information is handled through new user interaction metaphors.

The paper is organized as follows: In Section II, we explain all objectives, required functionalities and technological restrictions that guided this work. Section III contains the general system description including some implementation details. Furthermore, Section IV treats about special solutions to optimize the rendering capabilities through smart scene illumination and LoD (Level of Detail) management. It also focuses on the implementation of advanced features for user interaction and exploitation of semantic information.

\section{OBJECTIVES AND SYSTEM FunCTIONALITIES}

As has been mentioned before, the main objective of this work is the management of artistic information. But still hasn't been explained who the users are and which applications they could demand. One type of user actually are the artists: they rely on the existence of the system, and provide the contents to be managed and displayed. But the type of user really addressed by the virtual gallery is the "client", who could be any common internet user, a person of any age and education, having internet access of any type. All those different people should be attracted by a fast, comfortable and appealing application, something nowadays everybody is very accustomed to, and therefore is naturally demanded without taking into account the still existing technological restrictions. Hence, the most important 
requirement of this work is to build an efficient, low-cost application which is attractive through realistic visualizations. It also has to provide a flexible and fast interaction with user movements and information requests, and should be based on standard languages and public available stable plug-ins.

In the following, the main objectives of this work are listed within three groups: general system requirements, technical limitations to be overwhelmed and the inclusion of special advanced user interactive features.

\section{A. General system requirements}

a) Dynamic generation. The virtual environment has to be generated dynamically in the same moment a user wants to access it.

b) Visualization. Objects of interest, i.e. artworks, have to be presented in the virtual environment following specific visualization parameters which should include, among others, inter-object minimum distances, hanging positions and adequate illumination.

c) Adaptivity. The environment has to be generated adaptively to the content, i.e. to size and number of elements to be visualized.

d) Quick response to user actions. Visualization has to be adapted dynamically in response to the users' actions, i.e. showing better quality at a smaller viewing distance, by smartly using LoD and multiresolution management.

e) Navigation facilities have to be established inside the virtual scenarios to allow the users to move freely around the 3D scenes.

\section{B. Rendering and modelling language limitations}

There are several restrictions to handle with the VRML implementation language in the particular context of this application: limitations with the provided illumination facilities and limited abilities to manage the information associated to a given model. Therefore, the main technological problems to be solved are:

a) Efficient lightning management. Adaptation of the illumination to the avatars point of view inside the virtual environment. To allow proper rendering of the scene, the number of ligths has to be controlled. As the VRML base profile [2] establishes minimum requirements for the browsers, especially that at least 8 lights have to be rendered simultaneously, the application of more lights leads to undesired effects like saturation. Therefore, the designed architecture needs to adapt dynamically the number of lights relative to the avatar position and field of view.

b) Management of associated information. Artworks and other elements of the virtual environment may have multimedia information associated (i.e. explaining text, author information etc.) which strongly contributes to enrich the user's experience of the virtual gallery recreation. Access to this information has to be handled carefully, so that it could be:
- easily maintained (updated or modified) by artists, galleries or art foundations,

- filtered according to the user profile (e.g. language selection); and

- presented in an efficient way (offering the available information, but not rendering it until the user interaction with the virtual environment demands it).

\section{Advanced user interactive features}

The problems mentioned before should not be noticeable by the user; in contrary this third category treats about some very important objectives that require the implementation of advanced user based features.

a) User profile. Users should be able to select e.g. their preferred language for presentation of audio and textual information associated to the elements.

b) User interests. Users should be able to access the information of their interest in an effcient way, avoiding a possible overload caused by the presentation of less relevant information and allowing a smooth navigation inside the virtual world. This can be achieved by the design of a unique interaction interface which acts as an efficient hierarchical metaphor to show the information (such as audio, video, web pages, images or plain text) related to a particular work of art. Therefore, mechanisms to attach multimedia descriptions in the form of video or audio clips, text, WWW links, etc. should be provided and integrated into the 3D art gallery in a user-friendly way. In this way, the user could access this multimedia information by direct interaction with the art pieces included in the $3 \mathrm{D}$ scene.

\section{SYSTEM ARCHITECTURE}

In order to accomplish the formerly described goals, the following system architecture, based on a modular clientserver distribution like the one depicted in Fig. 1 is proposed.

The system workflow can be summarized as follows:

1) The content (artworks in our case) and its associated metadata are ingested in the system in a distributed way (provided by different sources, artists and art foundation, in the figure).

2) The scene description is generated by the Scene Description Generator (SDG), which creates an individual XML compliant description for every user, depending on their profile and interests.

3) Finally, the scene description is used to render the scene locally in the machine of the particular user who accesses to the system.

In the following subsections every module of this architecture will be describe in detail.

\section{B. Ingestion of content and metadata into the system}

The main characteristic of the system architecture is to allow the insertion of contents provided from different sources, following the Web 2.0 philosophy. This is achieved by defining a metadata schema (in our case XSD) to describe the characteristics of the added content. 


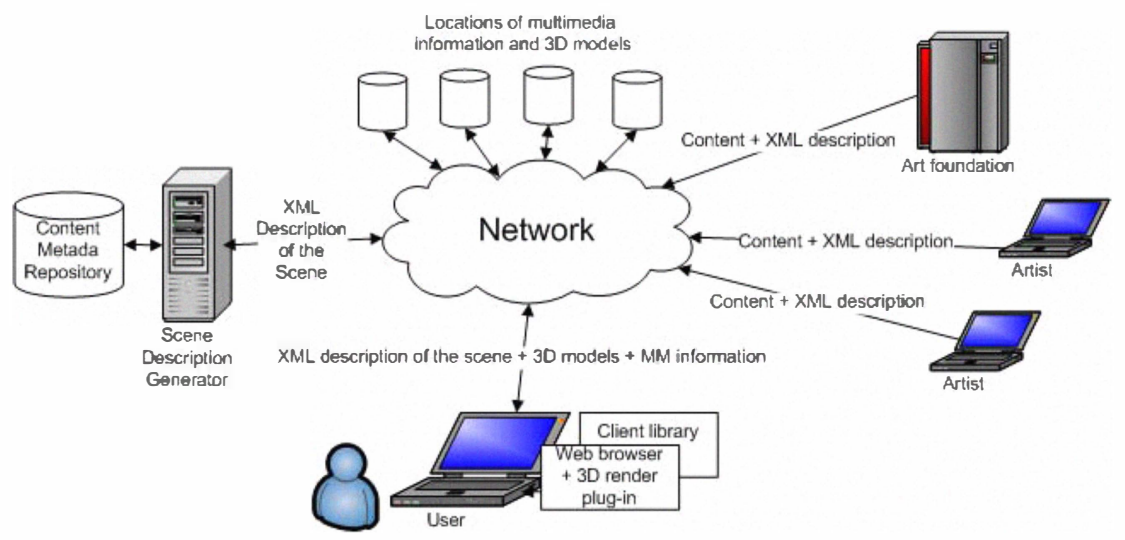

Figure 1. Distributed Client-Server System Architecture

The source providers could be anybody, e.g. artists themselves or art foundations (see Fig. 1). They introduce the content model, the related metadata description in XML format and the related multimedia information into the network. The content and the related multimedia data can be stored anywhere accessible in the network, and the content metadata description, in a Content Metadata Repository.

Every content 3D model could be as sophisticated as desired (i.e. a model of a sculpture), whereas the simplest one would be a rectangular canvas, in case that the provided content is a photo of a painting or an artistic photography. The exhibition space could also be a complex model (always having in mind that complex models need more rendering time) or just be created as rectangular room.

Each of the contributing entities can add contents to the system; they only need an application that formats the information according to the right scheme.

\section{Virtual scene XML description generation.}

The virtual Scene Description Generator (SDG in Fig. 1) creates sets of metadata in XML format [4] based on the following inputs:

- user interests: a list of artworks to be visualized, chosen by the user;

- user profile: the users' preferred language and their HW capabilities (e.g. bandwidth);

- metadata description of every object..

D. Dynamical generation of the virtual scene with adaptive rendering and visualization.

On the client side of the system, the XML description of the scene is processed by a client library (see Fig.1, below) which gives access to the necessary content models and their related information, and dynamically generate the virtual environment adapted to the user-selected content. Every model receives an appropriate illumination and an interaction metaphor which allows efficient user access to the related multimedia information

The user will be able to visualize and navigate through the virtual scene by using a standard web browser together with publicly available plug-ins (e.g. Cortona3D Viewer [5]).
Based on the description provided by that metadata set, the virtual gallery is dynamically generated. Particularly for canvases, they are placed throughout the walls keeping always minimum free spaces (defined by the content provider) between them (see snapshot in Fig. 2).

\section{Handling Of VRml Base Profile Limitations}

The VRML language has several advantages; it is standard and open, has publicly available stable plug-ins and establishes minimum requirements for browsers conforming to the VRML base profile [2], which is the commercially widest supported profile. As mentioned before, one of those requirements establishes a minimum number of 8 concurrent lights that have to be rendered simultaneously in a scene. Therefore, to avoid unexpected and undesired behaviors (like light saturation) in VRML base profile compliant browsers, the illumination models have to be managed intelligently to assure that the number of simultaneously illuminating lights is kept in a level supported by every browser. Particularly, the widely used Cortona3D Viewer [5], which was also used for our work, is VRML base profile compliant.

Besides, VRML also has limited abilities to efficiently manage information associated to a given model and to render it corresponding to specific user interaction.

Additionally, to improve rendering efficiency, a smart management of different model resolutions depending on the observation distances is required through the usage of LoDs (Levels of Detail). VRML does not define a normative way of manage them, but provides special tools for it [1].

In the following, the ways to overwhelm these limitations (LoD management, lightning and user interaction) will be described in detail.

\section{A. LoD (Level of Detail) management to adapt the virtual environment to the observation distance}

The most reasonable viewpoints in case of an art gallery are considered to be in front of the works of art. Here, the avatar is placed by default, i.e. at the beginning of the visit and when the user resets or recharges the scene. In case of paintings, the system renders medium resolution textures in the default positions for all canvases in the avatars field of view. The scene has to adapt dynamically when the user 
moves, offering higher quality recreations (rendering higher resolution textures when availabe) for detailed (closer) user observations and lower quality recreations for more global (further away) ones. For that purpose, we defined some distances $d_{n}$ (in $\mathrm{cm}$ ), where changes happen. Those distances depend on the number of different image resolutions provided in the metadata associated to the art work model, and are computed according to the following:

$$
d_{n}=d_{n-1}+2^{n-1} \cdot 0.5 \cdot \operatorname{dim}_{\max }
$$

where $n$ is the index of the texture of a picture, $\mathrm{d}_{0}=0$ (close to the wall) and $\operatorname{dim}_{\max }$ the size of the largest side of the painting (height or width). The smaller the observation distance of the avatar, the higher the resolution of the image, i.e. between $d_{0}$ and $d_{1}$ the highest resolution is presented, between $d_{1}$ and $d_{2}$ the second lower one and so on.

\section{B. Efficient lighting management.}

In arts, more than in any other area, the visual impression is of vital importance. It is well known, that the conditions of illumination have a great influence in the perception of objects. To simulate actual galleries illumination, lights should be equally distributed through the gallery, adapt to the dimensions of the canvases and their positions, and avoid saturation of the textures, which could happen when many lights are employed, as will be explained further on.

The VRML97 standard offers three shading algorithms of differing complexity: Flat-, Gouraud- and Phong-shading [2]. We decided to adopt Gouraud-shading, because Phongshading needs the highest calculation time and Flat-shading is incapable to create a nearly realistic environment. Nevertheless, it is necessary to compensate its limitations by

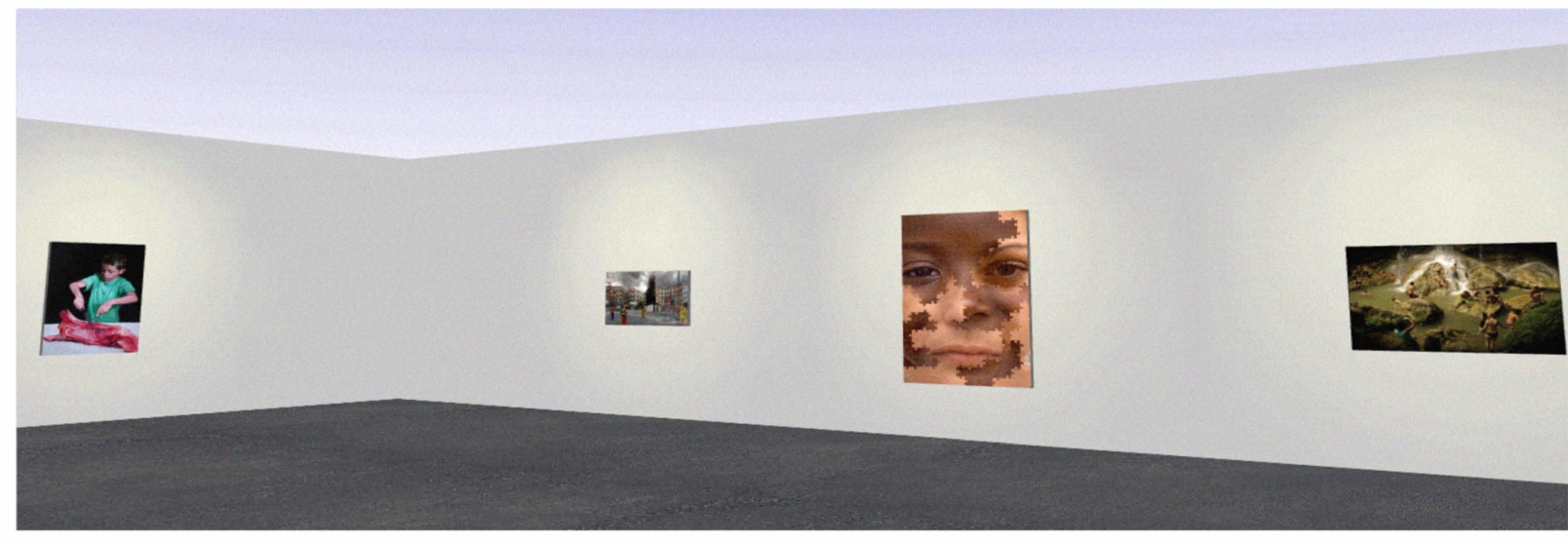

Figure 2. Rendering and Visualization of the Virtual Art Gallery

modeling flat surfaces with a high focus of attention using a variable number of triangles and thus reducing the number and size of uniformly illuminated areas. Particularly the walls require an efficient subdivision to avoid undesired jagging effects appearing in the scene. Here, polygon subdivision is applied selectively through the IndexedFaceset node [2].

Furthermore, VRML offers three types of lights, out of which we chose SpotLight: its name already indicates to be the most adequate one as it imitates best the spots used generally in art galleries.

The next important decision was the handling of lights per picture. For an efficient implementation, one spot is created per picture and installed at a reasonable illuminating distance. Nevertheless, to save execution time, not all spots are switched on during the whole time of visit through the gallery: only the objects falling into the field of view of the avatar are illuminated simultaneously. At this point, one of the VRML base profile requirements appears: when the field of view contains more artworks than the minimum number of lights simultaneously supported by the VRML base profile, the browser shows unrealistic and seriously disturbing visual effects of illumination saturation. To avoid these undesired effects, two main tradeoffs have been made: a) Reduction of the total number of spotlights in the scene. b) Adaptive reduction of the number of emitting spotlights relative to the movement of the avatar.

To resolve the first one, a new type of light has been introduced: the so called twin-light, which' task is to illuminate two artworks together. The idea is that not necessarily every two works of art share one light, only when they are placed near and they are small enough, they are candidates to be illuminated together by a twin-light. Twin-lights are of the same type as individual lights, they are only positioned between two objects and at a larger distance to ensure an adequate illumination of both.

The second tradeoff is achieved by introduction of a second level of lights, which, in opposite to the "normal" fixed positioned spotlights (first level), are temporarily created. Those temporary lights could be individual or twinlights, as the idea is to reduce the total number of lights adaptively to the movement of the avatar. Especially in scenes containing many artworks, the use of twin-lights would be very frequent, as the probability that too many objects are falling inside the field of view (and therefore the risk to create undesired effects like saturation) is quite high. At this point are operating two parallel algorithms:

\section{1) Flexible illumination handling.}

The objects which are not inside the viewing area of the avatar evidently do not need illumination. Therefore it is 
checked out continuously, which works of art are observed and the associated lights falling outside the viewing area are switched off temporarily, but not eliminated.

\section{2) Temporary light handling:}

When the illumination situation approximates to the limit number of $N$ spots turned on (where $N=8$ ), it is tested which are less relevant (the ones corresponding to pieces further away from the avatar) and could be substituted by temporal twin-lights. The original lights are not eliminated, only switched off, so the total number of emitting lights is $N-1$. This testing-routine works constantly, so that in the contrary case (no risk for saturation) the excessive temporary lights are eliminated. This real time adaptability of the scene illumination produces smooth lighting transitions which highly improve the visualization quality.

\section{User Interaction}

This work presents an efficient management of interactivity with the user, allowing dynamic adaptation of the visualization process in response to the users' actions in the virtual environment. We propose methods of user interaction which enhance significantly the comfort and usefulness of the tool, as they establish a direct relation to the users' profile and interests. This refers on one hand to the content shown in the gallery, which is completely selectable by the user, and on the other hand to the management of additional multimedia information associated to this content (i.e. the selected artworks).

\section{a) Gallery configuration}

The interaction with the user for the configuration of the gallery is established by using XML technology [4] with help of XML descriptions of the scene. The metadata related to every selected artwork is hierarchically structured with all the data necessary to generate a 3D object and the additional data for associated information. The data for every work of art description contains:

- Geometrical data. Dimensions of the art work and free space both sides.

- Identification data. Title and author of the artwork.

- Related multimedia information. Location of related text, audio, video, image and web sites.

- LoD and multi-resolution data. In case of paintings: images with up to four different resolutions.

All this information is used by the client library module (described in section III.D) to load the proper graphic models (canvases, painting or else), to access and download the required multimedia information and to dynamically manage the illumination model of the virtual scene.

b) Visualization of associated multimedia information

A flexible visualization of associated data is realized through a two-step interaction metaphor, which handles the information based on the XML description of the scene. This meta-information is made accessible through a menu, which automatically appears motionless on the upper part of the display when the user approaches a work of art to a certain minimum distance (first step). This menu, built of a template with up to five interactive elements (touch sensors) provide user access (second step) to an associated web-page, artwork information in text format, detail images, video or audio information. Fig. 3 shows the menu implemented in our application (gallery of paintings).

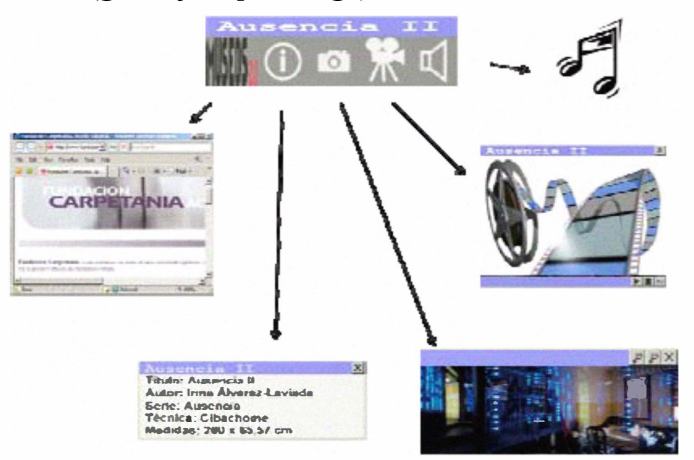

Figure 3. Multimedia Information Menu

\section{CONCLUSIONS}

In this paper, a VRML-based architecture for the automatic generation of user-customized virtual visits in 3D of art galleries based on XML descriptions is presented. The proposed objectives of general system requirements, as dynamic generation of the scene, adaptive visualization of the objects and integration of information management, have been successfully resolved by the implementation of a modular client-server architecture. Technical limitations like LoD and efficient lightning management have been overwhelmed, e.g. through the application of special lights (twin-lights). Furthermore, the inclusion of special advanced user interactive features has been achieved with help of interaction metaphors and decoupled XML descriptions carrying metadata, which is only displayed according to user requests. Summarizing, the proposed system architecture presents a very useful low-cost and platform-independent web application for the worldwide promotion of artists and their works.

\section{ACKNOWLEDGMENT}

This work has been supported by the Ministerio de Industria, Turismo y Comercio, plan AVANZA I+D, under project TSI-020302-2008-44 (Museos 2.0) and by the Ministerio de Educación y Ciencia under project TEC200767764 (SmartVision), both Ministries of the Spanish Government.

\section{REFERENCES}

[1] J. Hartman, J. Wernecke, "The VRML 2.0 Handbook" AddisonWesley, 1996.

[2] ISO/IEC 14772-1: "Virtual Reality Modelling Language" (VML97), 1997.

[3] ISO/IEC 19775: "Information technology - Computer Graphics and Image Processing - X3D", 2004.

[4] T. Bray, J. Paoli, C. M. Sperberg-McQueen, E. Maler, F. Yergeau. "Extensible Markup Language (XML) 1.0 (Fifth Edition)", W3C Recommendation, Nov. 2008.

[5] Parallel Graphics. “Cortona3D Viewer User's Guide”. June 2009. 